\title{
VAC_13 - Immunogenicity and safety of Yellow Fever Vaccine: systematic review and metanalysis
}

Paulo Roberto Gomes Takey ${ }^{*}$; Renata Saraiva Pedro ${ }^{1}$; Patrícia Mouta Nunes de Oliveira ${ }^{1}$; Letícia Kegele Lignani $^{1}$; Thalita da Matta de Castro ${ }^{1}$; Janaina Reis Xavier ${ }^{1}$; Maria de Lourdes de Sousa Maia ${ }^{1}$; Lusiele Guaraldo $^{2}$; Patricia Brasil ${ }^{2}$.

${ }^{1}$ Fiocruz/Bio-Manguinhos;

${ }^{2}$ Fiocruz/INI.

Introduction: Yellow fever is an acute febrile illness caused by the Yellow Fever Virus, which is mainly transmitted by mosquito bites in tropical and subtropical areas of Africa and South America. The change in epidemiology, risks of urban outbreaks and international spread, and consequent intermittent epidemics with significant morbidity and mortality rates, represent an emerging threat to Global Health. Vaccination remains the best strategy to eliminate yellow fever. However, we eventually face falls in coverage indicators caused by lack of access and vaccine hesitation during epidemics. Considering these issues, it is presented a systematic review and meta-analyzes of the evidence on immunogenicity and safety profile of yellow fever vaccine in fractional or full doses.

Objective: Systematize the evidence on immunogenicity and safety profile of yellow fever vaccine; Analyse the immunogenicity and adverse events following immunization; Estimate summary measures of immunogenicity and safety.

Methodology: A systematic review and meta-analyzes was conducted in accordance with PRISMA statement, in the second half of 2020. The bibliographic search of experimental and observational studies was made on comprehensive international databases, combining their descriptors and free terms in equations. The recovered articles were exported to Zotero for duplicates deletion, and then to Rayyan for consecutive and independent selection of titles, abstracts and texts. The evaluation of methodological quality of articles was carried out according to MINORS and RoB 2.0. The studies were described according to epidemiological design, immunogenicity, safety, period, population, and vaccine. The overall proportions were calculated based on proportions of seroconversion, and of serious and overall adverse events. Meta-analyzes were performed on R, based on the random effects model, and their results presented in forest plots.

Results: Of 1,724 scientific articles retrieved, 32 were selected: 23 observational and 9 experimental studies. The articles were published from 1999 to 2020, involved 15 to 1,171,889 participants of both sexes, from 9 months to 92 years of age, in countries of Americas, Africa, Asia and Europe, contemplating all vaccine producers in the world. The combined estimation for immunogenicity of full dose was $97 \%$ $(95 \% \mathrm{CI}=95 ; 98 \%)$, while for overall safety, $49 \%(95 \% \mathrm{CI}=40 ; 59 \%)$, exempt from vaccine-related serious adverse events. The combined estimation of immunogenicity and safety of yellow fever vaccine in fractional doses could not be calculated, since only one scientific article met the established inclusion criteria. But it ranged from 85,2 to $99,1 \%$ and 0 to $79,5 \%$, also exempt from vaccine-related serious adverse events, respectively.

Conclusion: The results of systematic review and meta-analyzes suggest using yellow fever vaccine, in fractional and full doses, as efficient and safe strategies for preventing disease. However, duration of immunity and risk factors of serious adverse events remain under discussion and are focused on ongoing and future studies.

Keywords: Immunogenicity; Safety; Yellow Fever Vaccine 